University of Wollongong

Research Online

Australian Institute for Innovative Materials -

Papers

Australian Institute for Innovative Materials

$1-1-2018$

Magnetoreresistance of carbon nanotube-polypyrrole composite yarns

R Ghanbari

Ferdowsi University of Mashhad

Shaban Reza Ghorbani

Ferdowsi University of Mashhad, ghorbani@uow.edu.au

H Arabi

Ferdowsi University of Mashhad

Javad Foroughi

University of Wollongong, foroughi@uow.edu.au

Follow this and additional works at: https://ro.uow.edu.au/aiimpapers

Part of the Engineering Commons, and the Physical Sciences and Mathematics Commons

Research Online is the open access institutional repository for the University of Wollongong. For further information contact the UOW Library: research-pubs@uow.edu.au 


\title{
Magnetoreresistance of carbon nanotube-polypyrrole composite yarns
}

\begin{abstract}
Three types of samples, carbon nanotube yarn and carbon nanotube-polypyrrole composite yarns had been investigated by measurement of the electrical conductivity as a function of temperature and magnetic field. The conductivity was well explained by 3D Mott variable range hopping (VRH) law at $\mathrm{T}<$ $100 \mathrm{~K}$. Both positive and negative magnetoresistance (MR) were observed by increasing magnetic field. The MR data were analyzed based a theoretical model. A quadratic positive and negative MR was observed for three samples. It was found that the localization length decreases with applied magnetic field while the density of states increases. The increasing of the density of states induces increasing the number of available energy states for hopping. Thus the electron hopping probability increases in between sites with the shorter distance that results to small the average hopping length.

\section{Disciplines}

Engineering | Physical Sciences and Mathematics

Publication Details

Ghanbari, R., Ghorbani, S. R., Arabi, H. \& Foroughi, J. (2018). Magnetoreresistance of carbon nanotubepolypyrrole composite yarns. Physica C: Superconductivity and its Applications, 548 78-81.
\end{abstract}




\title{
Magnetoresistance Behavior in Carbon-Nanotube Yarns
}

\author{
Ghanbari, Reza ${ }^{1,2}$; Ghorbani, Shaban Reza ${ }^{1,2, *}$; Arabi, Hadi ${ }^{1,2}$; Foroughi, Javad ${ }^{3}$ \\ ${ }^{l}$ Department of Physics, Faculty of Science, Ferdowsi University of Mashhad, Mashhad, Iran \\ ${ }^{2}$ Renewable Energies, Magnetism and Nanotechnology Lab. Faculty of science, Ferdowsi University of \\ Mashhad, Mashhad, Iran \\ ${ }^{3}$ ARC Centre of Excellence for Electromaterials Science, Intelligent Polymer Research Institute, University of \\ Wollongong, Wollongong, NSW 2519, Australia
}

\begin{abstract}
Carbon nanotube yarns are novel CNT-based materials that extend the advantages of CNT from the nanoscale to macroscale applications. Herein we have modeled the electrical properties of carbon nanotube yarn as a function of temperature and magnetic field. The conductivity was well explained by 3D Mott Variable Range Hopping (VRH) law at $T<100$ $\mathrm{K}$. Both the positive and negative magnetoresistance (MR) were observed at different magnetic field range. A quadratic positive MR was observed at small magnetic fields up to $B_{\text {tran, }}$, which is depended on temperature. It was found that the MR sign changed from positive to negative at $B_{\text {tran }}$. Then a quadratic negative MR was observed by increasing the magnetic field up to $B_{\mathrm{D} 2}$, where a deviation from quadratic magnetic field dependent of the MR was occurred. At intermediate magnetic field region, the negative MR shows linear magnetic field dependence up to magnetic field of $B_{\mathrm{D} 1}$. It was found that physical parameters such as the localization length and the density of states in Fermi level have a temperature and magnetic field dependent. In addition a general scaling behavior was found for the MR as function of $\left(B / B^{*}\right)^{1 / 3}$, where $B^{*}$ is a crossover magnetiic field,.
\end{abstract}

\section{1- Introduction}

Carbon nanotubes (CNTs) have been of great interest due to good electrical, chemical and mechanical properties. The CNTs show high electrical conductivity, thermal conductivity, chemical staibility and mechanical strength. These properties are suitable for a broad range of applications including electronic nano-devices, biosensors, chemical microsensors and energy storage. ${ }^{1-3}$ Recently, yarn microstructures were prepared in order to better using of the CNT features. ${ }^{4-7}$ It was found that the conductivity of CNT yarns are around $\sim 10^{4} \mathrm{~S} / \mathrm{cm}$ and $10^{2}-10^{3} \mathrm{~S} / \mathrm{cm}$ for single-walled carbon nanotube (SWCNT) yarns and multi-walled carbon nanotube (MWCNT) yarns, respectively. ${ }^{7,8}$

Transport mechanisms in CNT is most important for their performance improvements. ${ }^{9-}$ ${ }^{12}$ However, the undrestanding of conduction mechanisms has not yet completed even after years of research. ${ }^{13,14}$ This materials are neither fully amorphous nor completely crystalline, ${ }^{15}$ therefore the electrical transport in such disorder systems is described by a verity phenomena 
such as qusi-one dimensional transport, localization effects, hopping and tunlling transport ${ }^{16}$, ${ }^{17}$ and percolation. ${ }^{18,19}$ The conductivity of CNT yarn depend on the applying magnetic field. The magnetic field studies of the counductivity help investagators to more undrestanding of the electrons and spin transport.

The mechanisms of the MR phnamena in the organic systems including CNTs is explained by different models such as excitonic pair mechanism model, ${ }^{20}$ electron-hole recombination model, ${ }^{21}$ bipolaron model, ${ }^{22}$ forward interference model (also called orbital magnetoconductivity theory), and wave-function shrinkage model (WFSM). ${ }^{23}$ Here last two models will used to describe magnetoresistance of the CNTs yarn.

In this paper, CNT yarns were studied by measurement of the electrical conductivity as a function of temperature and magnetic field. The results shown that the temperature dependence of conductivity is well explained by 3D Mott-VRH model at $T<100 \mathrm{~K}$. Both positive and negative MR is observed at small magnetic fields and high magnetic fields, respectivly. Physical psrameters such as localization length, density of states and hopping average length were obtaained from the magnetic field dependence of the magnetoresistance. in addition, a scaling general behavior for MR was demonstrated.

\section{2- Experiment}

CNT Yarn was fabricated as described previously, ${ }^{4,24}$. The spinable multi walled carbon nanotube forest was synthesized by catalytic chemical vapor deposition (CVD) using acetylene gas as the carbon source. ${ }^{7}$ CNTs typically had diameters of about $10 \mathrm{~nm}$. The CNT yarns were drawn from the forest by pulling and twisting as described by Zhang et al. ${ }^{7}$

\section{3- Results and Discussion}

The tempereture dependence of the resistivity, $\rho(T)$, has been shown in the inset of Fig. 1 for the CNT yarn in the absence of a magnetic field. It can be seen that resistivity is 6 $\Omega . \mathrm{cm}$ at room temperature and it increase to $10.5 \Omega . \mathrm{cm}$ at $T=5 \mathrm{~K}$. 
In most of the heterogenous systems, which composed of partaily crystalline regions and disordered regions, the conductivity occur through tunnling and hopping. ${ }^{16,}{ }^{25}$ In this materials, charge carriers form polaron after doping and they are localized in gap region. ${ }^{26}$ The conduction show metalic behaviour in the crystalline regions and it occur in between two metallic domains through hopping ${ }^{26}$ (i.e. phonon-assisted tunneling between electronic localized states centered at different positions ${ }^{16}$ ). For these systems, the conductivity is well described by variable-range hopping model. ${ }^{27}$ The number of available energy states for hopping decrease with decreasing thermal energy $k_{\mathrm{B}} T$ and therefore hopping average length increases. ${ }^{16}$ This leads to the following expression for the conductivity ${ }^{27}$

$$
\sigma(T)=\sigma_{0} \exp \left[-\left(\frac{T_{M o t t}}{T}\right)^{\gamma}\right]
$$

where $\gamma=1 /(1+d)$ with $d=1,2,3$, which is called hopping dimensional. The pre-exponential $\sigma_{0}$ represents the conductivity at the high temperature limit and it is also temperature dependent but it is often neglected compared to the stronger temperature dependent of the exponential term. ${ }^{16,27} T$ is temperature and $T_{M o t t}$ is called the Mott characteristic temperature, which is related to the effective energy separations between localized states and can be expressed by following equation 27,28

$T_{\text {Mott }}=\frac{24}{\pi k_{B} N\left(E_{F}\right) \xi^{3}}$

where $N\left(E_{F}\right)$ is the density of states at the Fermi level, $\xi$ is the localization length of the wave function for the localized charge carrier, and $k_{\mathrm{B}}$ is Boltzmann constant.

The logarithm of conductivity was obtained based on Eq. 1 and it was plotted versus $T^{-1 /(1+d)}$ in Fig. 1. As can be seen in Fig. 1, evaluation of $\operatorname{Ln} \sigma v s . T^{-1 /(1+d)}$ with $d=1,2$ and 3 showed good fits to the experimental data only for $d=3$ at the temperature smaller than $T \approx 100 \mathrm{~K}$. Therefore, conductivity results are supported by the conductivity analyses in the frame of the three-dimensional (3D) variable range hopping (VRH) model at low temperature. While increasing temperatures causes a deviation from the 3D-VRH model. 


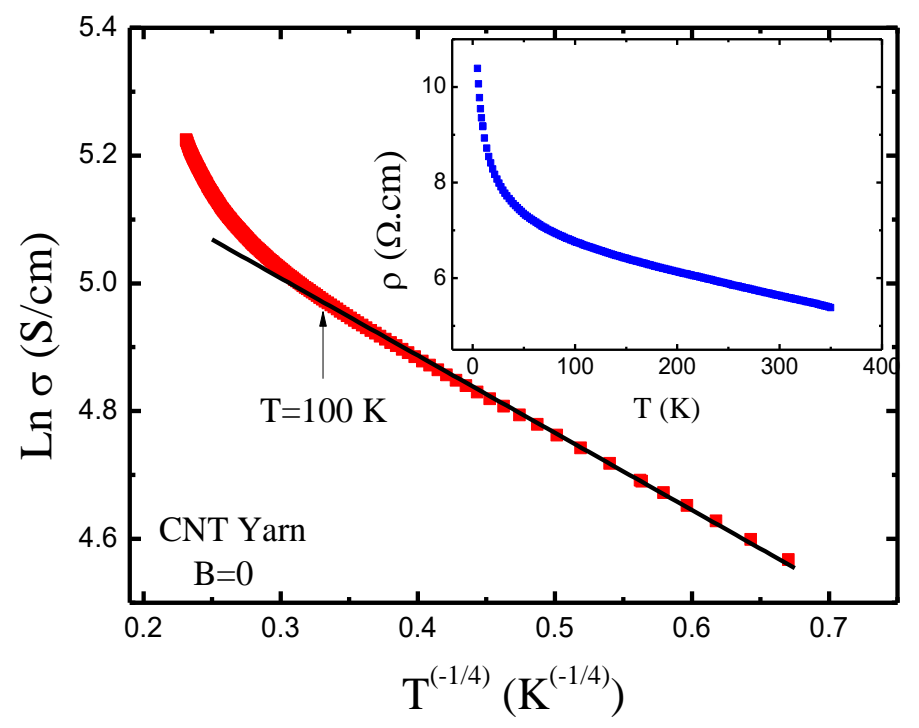

Fig. 1. Ln $\sigma v s . T^{(-1 / 4)}$ for CNT yarn in absence of a magnetic field, solid line shows the linear fitting for $T<100$ K. Inset: Temperature dependence of resistivity.

The Mott's characterization temperature $T_{M o t t}$ and $\sigma_{0}$ were obtained from the best fit of Eq. 1 with $d=3$ to experimental data. It was found the values of $2.14 \mathrm{~K}$ and $215.10 \mathrm{~S} / \mathrm{cm}$ for $T_{M o t t}$ and $\sigma_{0}$, respectively. These values will be used to calculate different parameters such as the localization length, the average hopping length, and density of states at Fermi level later. Gu et al. ${ }^{29}$ reported $T_{\text {Mott }}$ value of $17.75 \mathrm{~K}$ for MWCNT. As will discussed latter, generally, the smaller $T_{\text {Mott }}$ indicates a stronger localization of the charge carriers in the system, therefore, the results of the lower $T_{\text {Mott }}=2.14 \mathrm{~K}$ indicates weaker localization for CNT yarn. In fact, CNT yarns are made of a group of MWCNTs which are aligned, packed, and follow each other back to back using van der Waals interaction. Therefore, 3D-VRH conduction mechanism is dominated in disordered MWCNT. In this case, electrons can switch direction at the ends of every piece of MWCNT in the yarn and move to other piece of nearly MWCNTs, and it can explained 3D fitting model in conduction mechanism. ${ }^{4}$

The resistivity of materials can change under an external magnetic field. The magnetoreresistance is defined as $M R \%=[\rho(B, T)-\rho(0, T)] / \rho(0, T)^{*} 100$, where $\rho(0, T)$ and $\rho(B, T)$ are the resistivity without and under magnetic field $B$, respectively. The MR results of CNTs yarn is show in Fig. 2 at magnetic field of $0-10 T$ and different temperatures. 


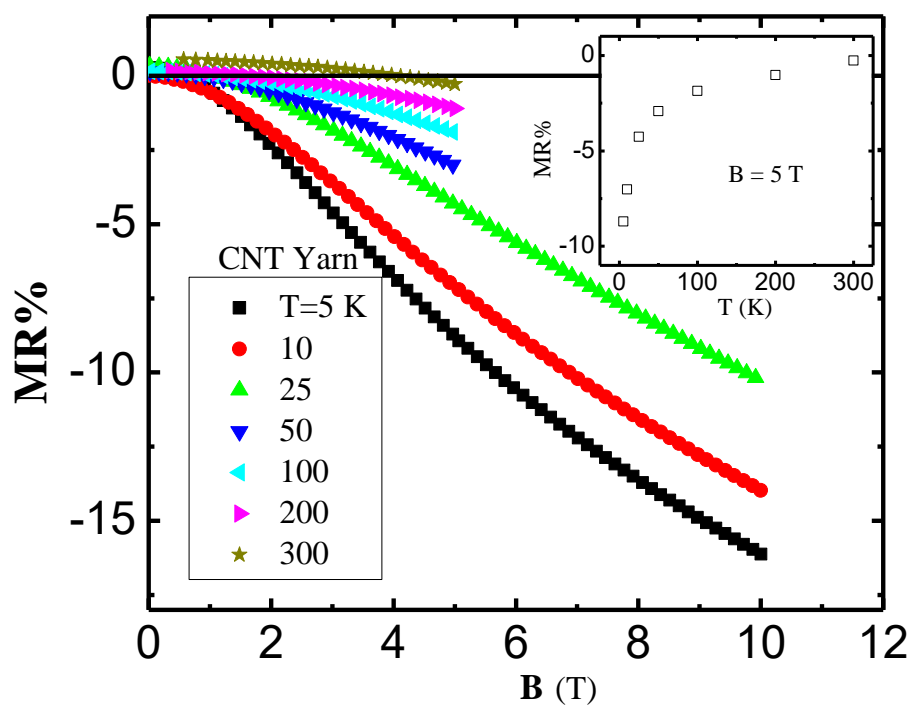

Fig. 2. Magnetic field dependence of the CNT yarn MR at different temperatures. Inset: The MR as a function of temperature in magnetic field of $5 \mathrm{~T}$.

Both positive and negative MR in different range of magnetic fields were observed. As can be seen in Fig. 2, there is a small positive MR at low magnetic field and it decrese to large negative values by increasing the magnetic field. The MR has been investigated for various kinds of CNT. ${ }^{30-32}$ Baumgartner et al. ${ }^{30}$ reported MR measurements on thin films of CNT. Their reported values of MR and the MR of CNT yarns have same order of magnitude, but the MR values of CNT yarns are less than CNT films. In additional, MR of CNT films was always negative (for temperatures above $4 \mathrm{~K}$ ) but both positive and negative MR were observed in CNT yarns. The MR of CNT yarns depend on temperature. Temperature dependence of the MR is shown in inset of Fig. 2. It increases with increasing temperature. At magnetic field of $5 \mathrm{~T}$, the MR value is -8.7 at temperature of $5 \mathrm{~K}$ and it increase to -1.1 for temperature of $200 \mathrm{~K}$.

Generally, the negative MR is observed in materials located on the metallic side of the metal-insulator transition (MIT), which is explained by considering quantum corrections due to the weak localization and electron-electron interactions. ${ }^{33,34}$ In the insulator side and near the MIT, the negative MR is explained based on the manifestation of the quantum interference among random paths in the hopping process. ${ }^{33}$, 35 This interference was increasing the hopping probability therefore results to decrease the resistivity and appearing 
the negative MR. While the positive MR of materials, which are in the insulator side of the MIT, is due to hopping mechanism of conduction. It was found that the positive MR varies exponentially with magnetic field. ${ }^{36}$ This behavior of the positive MR was explained by squeezing of the wave functions of impurity electrons in the transverse direction of magnetic field. It leads to a sharp decrease in the overlap of the wave function of neighboring impurities results to decreasing the hopping probability between two sites results to an exponentially increased resistivity with increasing magnetic field and causes a positive MR. ${ }^{36}$, 37 It was reported that external magnetic fields affected on localization and electron correlation. ${ }^{38}$ The contribution to the magnetoresistance due to electron-electron interaction is positive while the sign of the magnetoresistance associated with localization can be negative or positive depending on spin-orbit effects. ${ }^{39}$ It was found that a weak spin-orbit effect at present magnetic field gives a negative MR ${ }^{40}$ while strong spin-orbit scattering is induced a positive MR. ${ }^{41}$ Our results show that the electron correlation is not important in these materials due to a negative MR and that localization is due to weak spin-orbit scattering. Both the positive and negative MR will discuss in more detail below.

\section{A. Positive Magnetoresistance Theory: The wave function shrinkage model}

Strong increasing of the positive resistance (or the positive MR) with a magnetic field had been predicted originally by Tokumoto et al., ${ }^{42}$ and Shklovskii, ${ }^{43}$ until it was elaborated by Shklovskii and Efros using the wave function shrinkage model. ${ }^{36,}{ }^{44}$ According to this model the magnetic field decrease overlap probability of the impurities electron wave function between two sites that increase the resistivity in magnetic field. This model predicts two regions for the positive MR in the Mott-VRH regime that they depend on magnetic field.

At the weak magnetic field, the resistivity at magnetic field of $B$ and temperature of $T$, $\rho(B, T)$, is described by the following expression 25,36

$$
M R=\frac{\Delta \rho(B, T)}{\rho(0, T)} \approx t \frac{e^{2} \xi^{4}}{36 \hbar^{2}} B^{2}\left[\frac{T_{M o t t}}{T}\right]^{\frac{3}{4}}
$$

where $T_{M o t t}$ and $\xi$ are characteristic Mott temperature and localization length, respectively, and $t=5 / 2016$ is a numerical constant. $\rho(0, T)$ is the resistivity in the zero magnetic fields and temperature of $T$, which is obtained from inverse of Eq. 1 in the frame of the threedimensional (3D) VRH model. $e$ and $\hbar$ are electron charge and the reduced Plank's constant, 
respectively. While at the strong magnetic field, Shklovskii and Efros ${ }^{36}$ showed different magnetic field and temperature dependence for the resistivity as ${ }^{33}$

$$
\operatorname{Ln}\left(\frac{\rho(B, T)}{\rho(0, T)}\right) \propto \xi^{\frac{2}{3}} B^{\frac{1}{3}}\left[\frac{T_{\text {Mott }}}{T}\right]^{\frac{1}{3}}
$$

This field dependence $\left(B^{1 / 3}\right)$ for positive MR was observed in CNT/Polyaniline composite film at very low temperatures. ${ }^{45}$

Fig. 3 show MR\% versus $B^{2}$ at the different temperatures. It can be seen that MR decrease linearly in the positive MR region for three different temperatures and it is well described by Eq. 3. In addition with increasing magnetic field, the MR transition from

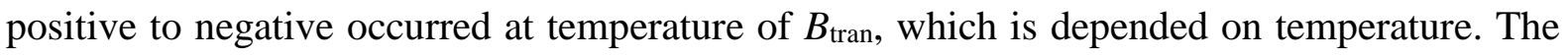
transition field is about $0.5,1.0$ and $0.8 \mathrm{~T}$ at temperature of 5,25 and $50 \mathrm{~K}$, respectively.

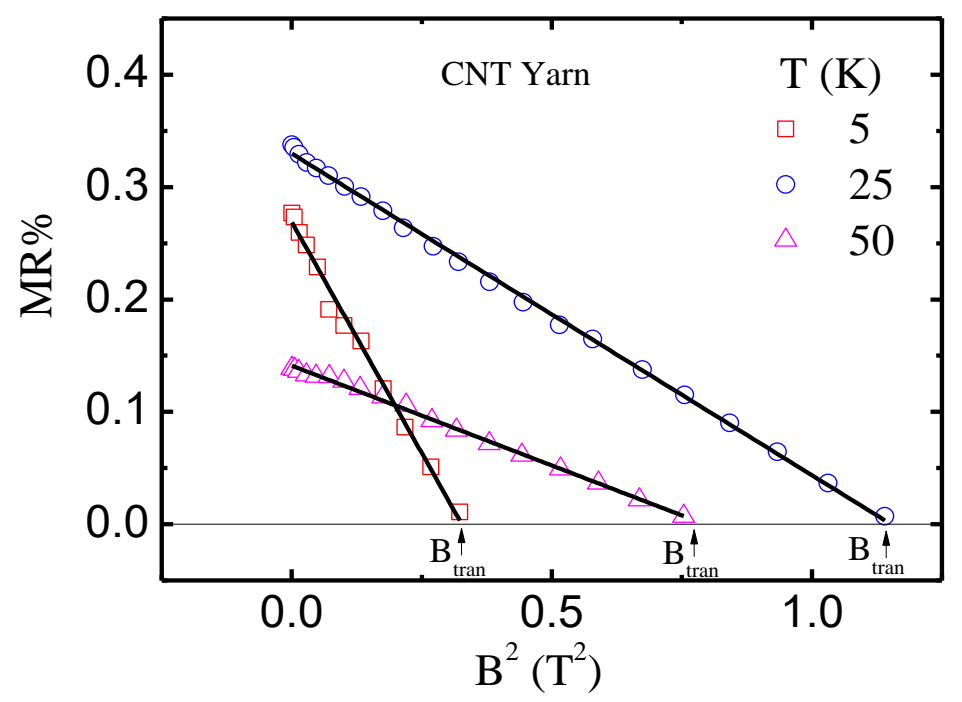

Fig. 3. MR\% vs. $B^{2}$ in the positive MR region at the different temperatures. Solid lines show the best fit of Eq. 3 to experimental data. $B_{\text {tran }}$ show MR transition from positive to negative.

The localization length, $\xi$, can be obtained from Eq. 3 by the following expression:

$$
\xi^{4}=\frac{36 \hbar^{2}}{t e^{2}} M R\left[\frac{T}{T_{\text {Mott }}}\right]^{\frac{3}{4}} B^{-2}
$$

The localization length was obtained based on Eq. 5 by using the calculated values of $T_{M o t t}=2.14 \mathrm{~K}$ and the normalized resistivity in the positive MR region. Results of the 
localization length (in the positive MR region) as a function of magnetic field have been plotted in Fig. 4(a) for CNT yarn at the different temperatures by open symbols. It can be seen in Fig. 4(a) that the localization length is approximately 500 and $800 \mathrm{~nm}$ at temperatures of 5 and $25 K$, respectively, at magnetic field of $\approx 0.02 \mathrm{~T}$, which is in agreement with the order of measured 1D localization length of mats of SWCNT. ${ }^{32}$ This long localization length can be due to coherency distortion by electron-phonon scattering. ${ }^{32}$ Also, it can be seen that the localization length decreases with applying the magnetic field. This behavior can be due to squeezed of the wave function of the impurity electrons under the applied magnetic field.

The hopping average length, $R_{h o p}$, in Mott's VRH regime is given by: ${ }^{44}$

$R_{\text {hop }}=\left(\frac{3}{8}\right)\left(\frac{T_{\text {Mott }}}{T}\right)^{\gamma} \xi$

From the obtained results for the localization length in the Fig. 4(a), the average hopping length and the density of states in the Femi level, $N\left(E_{F}\right)$, are obtained by using the Eqs. 2 and 6. Fig. 4(b) shows the $N\left(E_{F}\right)$ for the positive MR region as function of magnetic field for the CNT yarn at the different temperatures that are shown by open symbols. The density of states of electron observed by Tsebro et al. ${ }^{46}$ for MWCNTs was $\sim 10^{18}\left(\mathrm{eV} . \mathrm{cm}^{3}\right)^{-1}$, ${ }^{47}$ while the values observed for CNT yarn are $3.2 \times 10^{17}$ and $\left.8.5 \times 10^{16}\left(\mathrm{eV}^{\mathrm{cm}}\right)^{3}\right)^{-1}$ at temperatures of 5 and $25 \mathrm{~K}$, respectively, at magnetic field of $\approx 0.02 \mathrm{~T}$. As can be seen in Fig. 4(b), the density of states at the Fermi level has increased with increasing magnetic field. At magnetic field of 0.5 and $1.0 \mathrm{~T}$, the density of states values are $4.6 \times 10^{20}$ and $6.7 \times 10^{20}\left(\mathrm{eV} . \mathrm{cm}^{3}\right)^{-1}$ at temperature of 5 and $25 \mathrm{~K}$, respectively. As discussed above, the wave function of charge carriers was squeezed and results to decreasing of the localization length with applied magnetic field therefore new states was created around the Femi level results to enhance the density of states.

Fig. 4(c) shows the average of the hopping length, $R_{h o p}$ in the positive MR region versus magnetic field at the different temperatures, which were shown by open symbols for CNT yarn. It can be seen that the $R_{h o p}$ decreases with increasing of the magnetic field. Since the increasing of the density of states results to increasing of the number of available energy states for hopping. Therefore the electron hopping probability increases due to the shorter distance between states and thus the average hopping length decreases. This result to increase the electrical resistivity and decreases the rate of the average hopping length, which was caused to the positive MR, with increasing the magnetic field. 

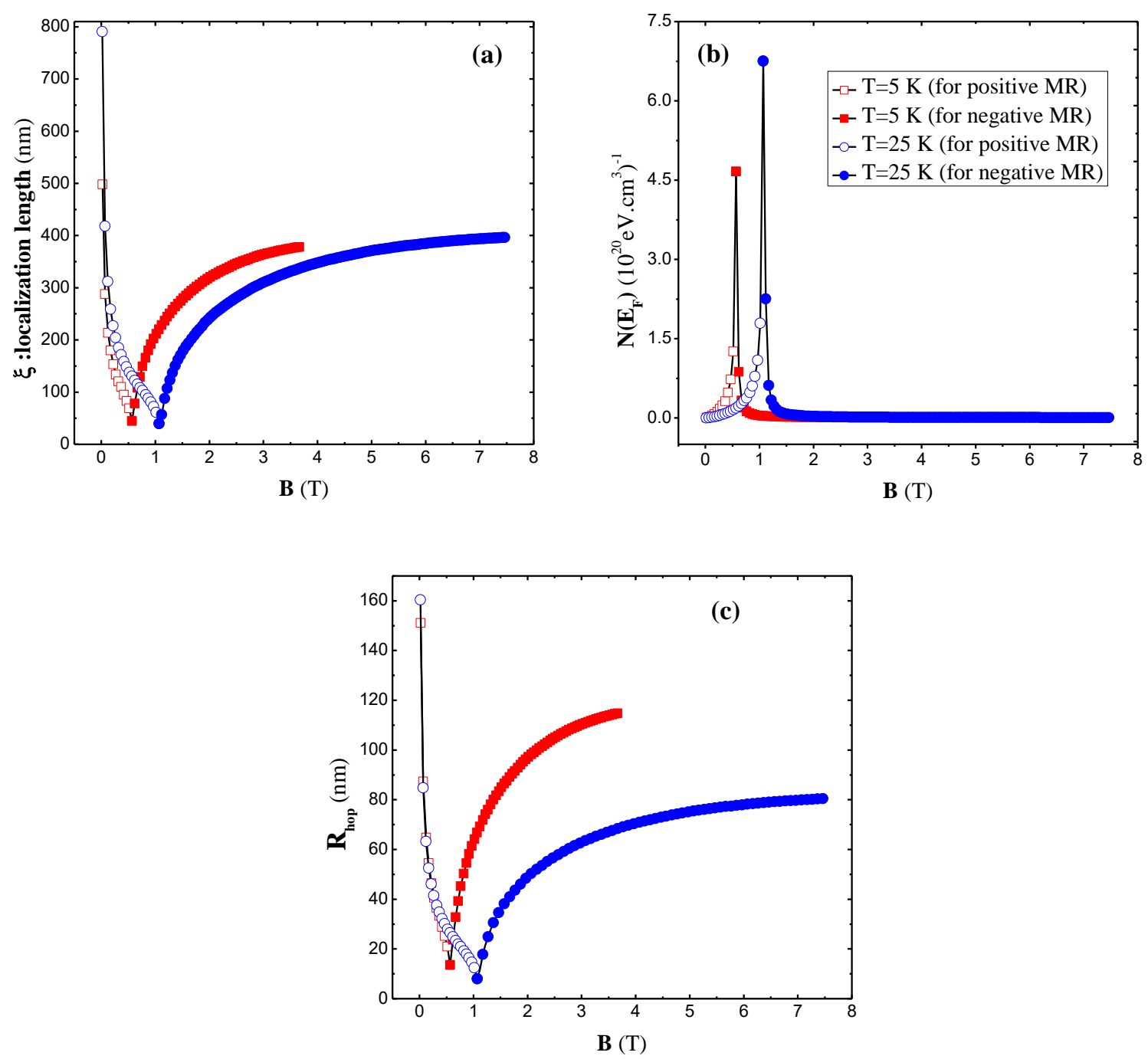

Fig. 4. The physical parameters for positive (open symbols) and negative (solid symbols) MR region as a function of the magnetic field at temperature of 5 and $25 \mathrm{~K}$ for CNT yarn. (a) The localization length $\xi$. (b) Density of states in Fermi level $N\left(E_{F}\right)$. (c) Average hopping length, $R_{\text {hop }}$.

As can be seen in Fig. 2, the MR shows a transition from the positive to the negative MR. Although the electrical resistivity increased with applied a constant magnetic field in the positive MR region, but it decreases by increasing of magnetic field in this region, i.e. $d \rho / d B<0$. Thus the MR decreases with increasing the magnetic field until it becomes zero and then it became negative values. This means that the MR mechanism change and therefore another mechanism will induce the negative MR. 


\section{B. Negative Magnetoresistance Theory: The Forward Interference Theory}

The forward interference theory or orbital magnetoresistance theory, which was proposed by Nguyen, Spivak, and Shklovskii (NSS), ${ }^{48}$ predict a small negative MR., In this model, the effect of forward interference of random and different paths are considered in the hopping process between two sites at an average distance of $R_{h o p}$. According model, interference between all of the possible hopping paths between sites of 1 and 2 within a cigar-shaped domain with length of $R_{h o p}$ and width of $\left(\xi R_{h o p}\right)^{1 / 2}$ will influence on hopping probability between two sites. By the numerically logarithm averaging of the conductivity over many different possible paths in the present of the magnetic field, they obtained a linear negative MR in the low magnetic field. While based on the NSS model and using a critical percolation method instead of logarithmic averaging method, a quadratic magnetic field dependence for the negative MR was also obtained at small magnetic fields: ${ }^{33,35}$

$$
\left(\frac{\Delta \rho}{\rho}\right)_{-} \propto T^{-\frac{3}{4}} B^{2}
$$

It was found ${ }^{35}$ that the quadratic magnetic field dependence of the MR is extremely small and at higher magnetic fields there is a deviation from this behavior at magnetic field of $B_{\mathrm{D} 2}$. At magnetic field larger than $B_{\mathrm{D} 2}$, the resistivity ratio $\rho(B, T) / \rho(0, T)$ can be expressed by approximately the following expression: ${ }^{49}$

$$
\frac{\rho(B, T)}{\rho(0, T)} \approx 1-C_{\text {sat }} \frac{B}{B_{s a t}(T)}
$$

So $\mathrm{MR} \approx-C_{\text {sat }} B / B_{\text {sat }}$, where $C_{\text {sat }}$ is a temperature independent saturation constant and $B_{\text {sat }}$ is the effective saturation magnetic field, which depend on temperature. Based on the MottVRH electrical conduction mechanism, the $B_{\text {sat }}$ parameter is given by following expression: ${ }^{37}$, 50

$$
B_{\text {sat }} \approx 0.7\left(\frac{8}{3}\right)^{\frac{3}{2}}\left(\frac{h}{e}\right)\left(\frac{1}{\xi}\right)^{2}\left(\frac{T}{T_{\text {Mott }}}\right)^{\frac{3}{8}}
$$

Fig. 5 shows the negative MR as a function of magnetic field at three different temperatures for the CNT yarn. As can be seen in the Fig. 5, there are three different regions. 
At small magnetic field, which is shown in the large scale in the inset of Fig. 5 for clarifying, the Eq. 7 well describe experimental data up to magnetic field of $B_{\mathrm{D} 2}$, where there is a deviation from its. At intermediate magnetic field region, the data shows a linear magnetic field dependence up to magnetic field of $B_{\mathrm{D} 1}$. In this region experimental data of the negative MR was well described by Eq. 8 at magnetic fields lower than $B_{\mathrm{D} 1}$, as shown by the solid curves in Fig. 5. Linear magnetic field dependence has been observed in CNT/polyaniline composite films at intermediate magnetic field region. ${ }^{45}$ The best-fitted value of $C_{\text {sat }}$ is 0.2 and $B_{\text {sat }}$ are 9, 11 and $15 T$ at temperature of 5, 10 and $25 \mathrm{~K}$, respectively, for the CNT yarn. While at the magnetic field larger than $B_{\mathrm{D} 1}$, the Eq. 8 cannot describe experimental data.

By using values of the fitting parameter of $B_{\text {sat }}$ and $T_{M o t t}=2.14 \mathrm{~K}$, the localization length $\xi$, the density of states at Fermi energy $N\left(\mathrm{E}_{\mathrm{F}}\right)$ and the hopping average length $R_{h o p}$ were obtained by using the equations of 9,2 and 6, respectively, in the negative MR region. Results of these physical parameters have been shown in Fig. 4 by solid symbols for the negative MR region for two different temperatures. As can be seen in Fig. 4, the trend of these physical parameters at the positive and negative MR regions are quite different. In the positive region, both parameters of the $\xi$ and the $R_{h o p}$ decrease but the $N\left(E_{F}\right)$ increase with increasing magnetic field. While in the negative region, these parameters increases with increasing magnetic field and becomes constant at the higher magnetic fields. As it mentioned, by applying magnetic field, overlap probability of the impurities electron wave function decrease and localization be stronger. But, by increasing magnetic field, the interference effect, referred as a weak localization, grows. ${ }^{51}$ The magnetic field reduces coherent backscattering in disordered system such as CNT yarn because carriers acquired an additional phase in moving paths. ${ }^{51}$ Electron-phonon and electron-electron interactions are the main decoherence mechanisms at low temperatures. ${ }^{47}$ There is a completion between these two effects as weak localization effect gets stronger with magnetic field increasing. Therefore, localization length has a minimum when these two effects are equal contributions


maximum at $B_{\text {tran }}$. 


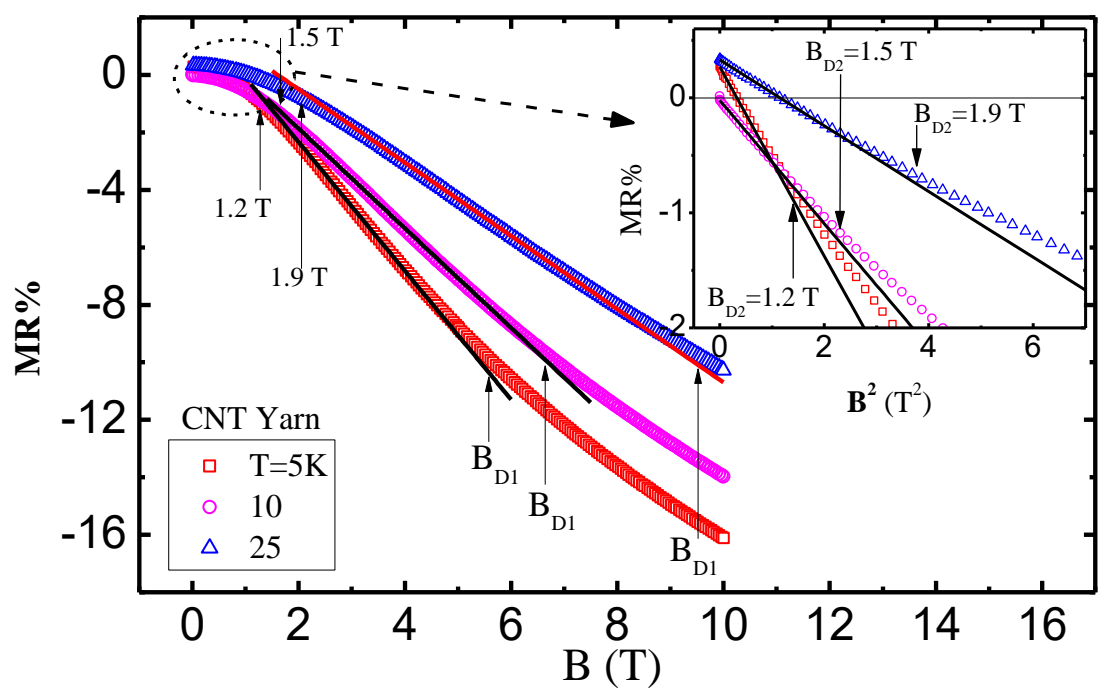

Fig. 5. The MR\% vs. magnetic field. There are three different regions. Arrows show deviation from magnetic field dependence of the negative MR at each region. The first regions are shown in larger scale in inset for clarifying.

As shown in Fig. 5, the linear dependence of the MR is deviated at the magnetic field of $B_{\mathrm{D} 1}$. For investigation of the MR at higher magnetic fields, we repotted the CNT Yarn MR data $v$ s. $B^{1 / 3}$ in Fig. 6 for different temperatures. It can be clearly seen that the negative MR is in close agreement with the behavior $B^{1 / 3}$ in the third region. As can be seen in Fig. 6, the CNT Yarn MR data shows at least one crossover field such as $B^{*}$, which is shown by arrows. The crossover field $B^{*}$ depend on temperature and it increase with increasing temperature. The existance of crossover show a transition in the mechanism of the MR. The MR is approximatly $B^{1 / 3}$ independent when the applied magnetic field is lower than the crossover field $B^{*}$. 


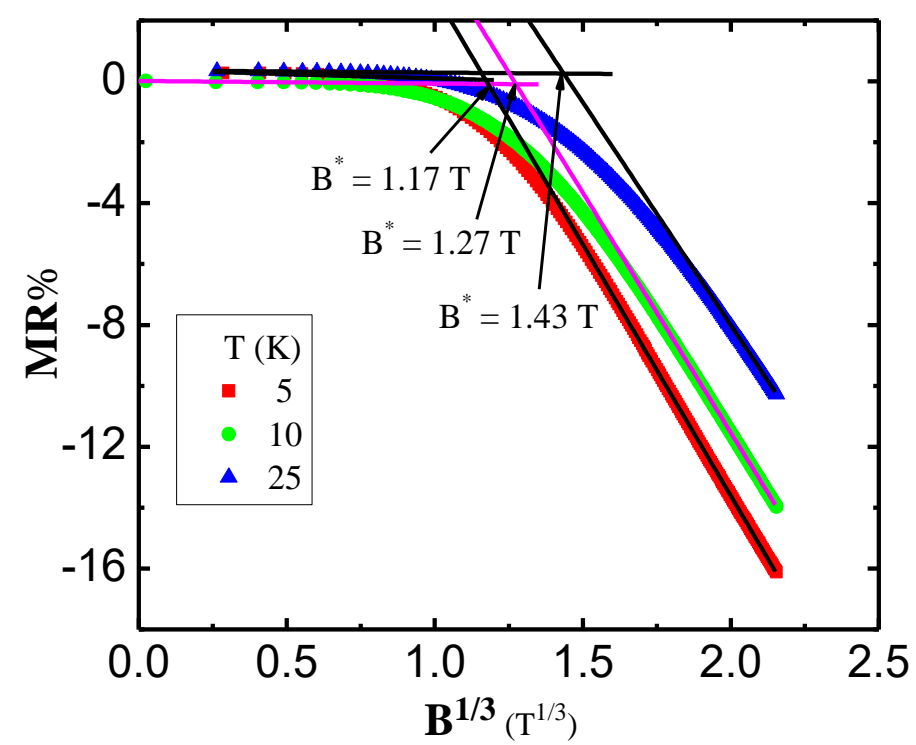

Fig. 6. MR\% vs. $B^{1 / 3}$ for CNT yarn at different temperatures. The crossover magnetic field $B^{*}(T)$ was shown by arrows.

The scaling behavior for the MR was examined for $B / B^{*}$, where $B^{*}$ is the crossover magnetic field. The results are shown in Fig. 7. As can be seen in Fig. 7, the MR at fields, which is studied here, has been scaled into one curve with the obtained the crossover magnetic field. A further study is needed to elucidate the origin of this scaling behavior.

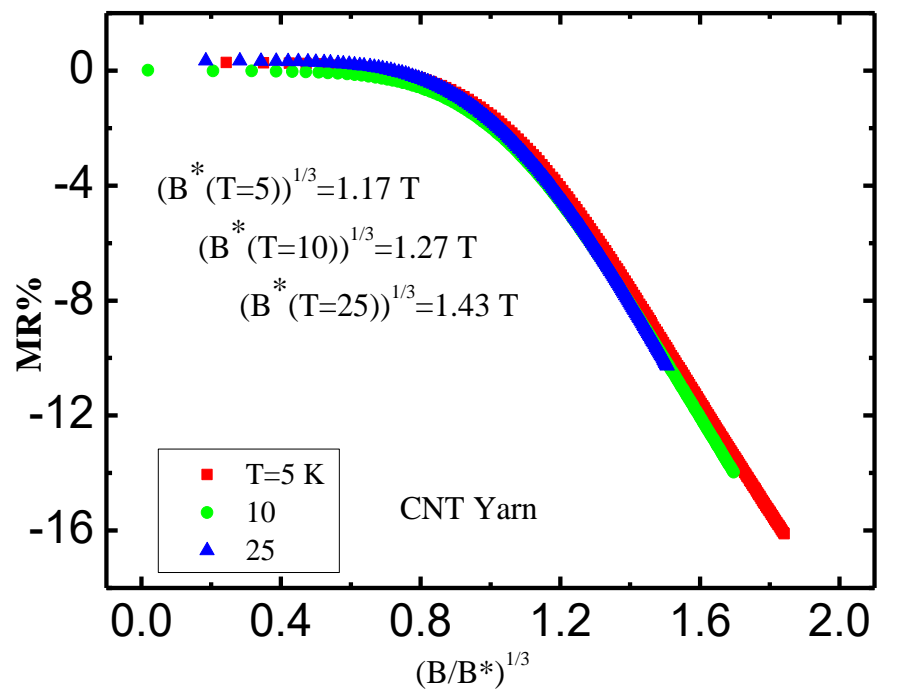

Fig. 7. Scaling behavior for the MR\% vs. $\left(B / B^{*}\right)^{1 / 3}$ for CNT yarn at different temperatures. 


\section{4- Conclusion}

The resistivity of CNT Yarn increases with decreasing of temperature. The best fitting of conductivity in hopping conduction mechanism obtained for $d=3$ at the temperatures down to $T \approx 100 \mathrm{~K}$, thus it indicate that the conduction mechanisms correspond to a 3D Mott VRH model for $T<100 \mathrm{~K}$. A small positive MR observed at low magnetic field and it reached to large negative values by increasing the magnetic field. The quadratic positive MR observed at small magnetic fields which it is well explained by Wave Function Shrinkage Model. The wave function of charge cariers was squeezed and leads to decreasing of the localization length with applied magnetic field and density of states in Fermi level increases. The increasing of the density of states means increasing of the number of available energy states electrons for hopping, the electron hopping probability with shorter distances increases, thus the average hopping length decreases. The quadratic negative MR observed at low magnetic field which deviated at deviation magnetic field $B_{\mathrm{D} 2}$.With increasing of the magnetic field a linear negative MR is observed which it is deviated at the deviation magnetic field $B_{\mathrm{D} 1}$. The both quadratic and linear negative MR are well explained by Forward Interference Theory. Also, it observed that localization length of charge carriers has a minimum with magnetic field which is temperature dependent. Also, the density of states in Fermi level has a maximum with magnetic field; therefore, hopping average length has a minimum. Also, the negative $\mathrm{MR}$ is in close agreement with the behavior $B^{1 / 3}$ at higher magnetic fields for different temperatures and MR data shows at least one crossover field such as $B^{*}(\mathrm{~T})$. Also, it was observed a general scaling behavior for the MR as function of $\left(B / B^{*}\right)^{1 / 3}$.

\section{Acknowledgements}

The authors acknowledge funding from the Ferdowsi University of Mashhad (Grant no. 3/42918). The authors would like to thank the Australian Research Council under Discovery Early Career Researcher award (J. Foroughi DE12010517) and also thank Prof. R.

Baughman, University of Texas at Dallas, for his continuous support, insights, and also for providing the CNT forests needed for producing the yarns.

\section{Reference}

P. L. McEuen and J.-Y. Park, MRS Bulletin 29, 272 (2004).

J. Foroughi, et al., Science 334, 494 (2011).

H. Dai, Accounts of Chemical Research 35, 1035 (2002). 
J. Foroughi, B. Kimiaghalam, S. R. Ghorbani, F. Safaei, and M. Abolhasan, Thin Solid Films 520, 7049 (2012).

J. Foroughi, et al., Advanced Functional Materials 24, 5859 (2014).

J. Foroughi, G. M. Spinks, S. R. Ghorbani, M. E. Kozlov, F. Safaei, G. Peleckis, G. G. Wallacea, and R. H. Baughmanc, Nanoscale 4, 940 (2012).

M. Zhang, K. R. Atkinson, and R. H. Baughman, Science 306, 1358 (2004).

L. Zhu, J. Xu, Y. Xiu, Y. Sun, D. W. Hess, and C.-P. Wong, The Journal of Physical Chemistry B 110, 15945 (2006).

A. I. Hochbaum, R. Chen, R. D. Delgado, W. Liang, E. C. Garnett, M. Najarian, A. Majumdar, and P. Yang, Nature 451, 163 (2008).

B. Poudel, et al., Science 320, 634 (2008).

D. Bhattacharyya, R. Yang, and K. K. Gleason, Journal of Materials Chemistry 22, 17147 (2012).

T. Takano, H. Masunaga, A. Fujiwara, H. Okuzaki, and T. Sasaki, Macromolecules 45, 3859 (2012).

C. Yong, M. T. George, S. Paul, and J. H. Alan, Applied Physics Letters 60, 2711 (1992).

N. S. Sariciftci, A. J. Heeger, and Y. Cao, Physical Review B 49, 5988 (1994).

A. Ugur, F. Katmis, M. Li, L. Wu, Y. Zhu, K. K. Varanasi, and K. K. Gleason, Advanced Materials 27, 4604 (2015).

A. B. Kaiser, Rep. Prog. Phys. 64, 1 (2001).

O. Bubnova, et al., naturematerials 13, 190 (2013).

Y. Long, Z. Chen, X. Zhang, J. Zhang, and Z. Liu, J. Phys. D: Appl. Phys. 37, 1965 (2004).

W. Bauhofer and J. Z. Kovacs, Composites Science and Technology (2008).

Y. Sheng, T. D. Nguyen, G. Veeraraghavan, O. Mermer, M. Wohlgenannt, S. Qiu, and U. Scherf, Physical Review B 74, 045213 (2006).

V. N. Prigodin, J. D. Bergeson, D. M. Lincoln, and A. J. Epstein, Synthetic Metals 156, 757 (2006).

W. Wagemans, F. L. Bloom, P. A. Bobbert, M. Wohlgenannt, and B. Koopmans, Journal of Applied Physics 103, 07F303 (2008).

H. Haken and H. C. Wolf, The physics of atoms and quanta: introduction to experiments and theory (Springer Science \& Business Media, 2005).

J. Foroughi, G. M. Spinks, S. R. Ghorbani, M. E. Kozlov, F. Safaei, G. Peleckis, G. G. Wallace, and R.

H. Baughman, Nanoscale 4, 940 (2012).

H. Gu, J. Guo, X. Yan, H. Wei, X. Zhang, Y. Huang, S. Wei, and Z. Guo, Polymer 55, 4405 (2014).

S. Bhadra, D. Khastgir, N. K. Singha, and J. H. Lee, Progress in Polymer Science 34, 783 (2009).

F. N. Mott and E. A. Davis, Electronic Processes in Non-Crystalline Materials (Clarendon, Oxford, 1979).

L. Zhang and Z.-J. Tang, Physical Review B 70, 174306 (2004).

H. Gu, et al., Nanoscale 6, 181 (2014).

G. Baumgartner, M. Carrard, L. Zuppiroli, W. Bacsa, W. A. de Heer, and L. Forr ${ }^{3}$, Physical Review B 55, 6704 (1997).

Y. Yosida and I. Oguro, Journal of Applied Physics 86, 999 (1999).

M. S. Fuhrer, W. Holmes, P. L. Richards, P. Delaney, S. G. Louie, and A. Zettl, Synthetic Metals 103, 2529 (1999).

L. Essaleh and S. M. Wasim, in Smart Materials for Energy, Communications and Security (Springer, 2008), p. 127.

A. Kawabata, Solid State Communications 34, 431 (1980).

U. Sivan, O. Entin-Wohlman, and Y. Imry, Physical Review Letters 60, 1566 (1988).

B. I. Shklovskii and A. L. Efros, Electronic Properties of Doped Semiconductors (Springer-Verlag, New york Tokyo, 1984).

H. Gu, J. Guo, X. Yan, H. Wei, X. Zhang, Y. Huang, S. Wei, and Z. Guo, Polymer 55, 4405 (2014).

P. A. Lee and T. V. Ramakrishnan, Reviews of Modern Physics 57, 287 (1985).

P. Dai, Y. Zhang, and M. P. Sarachik, Physical Review B 46, 6724 (1992).

A. Kawabata, Journal of the Physical Society of Japan 49, 628 (1980).

S. Hikami, A. I. Larkin, and Y. Nagaoka, Progress of Theoretical Physics 63, 707 (1980).

H. Tokumoto, R. Mansfield, and M. J. Lea, Philosophical Magazine Part B 46, 93 (1982).

B. I. Shklovskii, Zh. Eksp. Teor. Fiz. 36, 43 (1982).

R. Rosenbaum, T. Murphy, E. Palm, S. Hannahs, and B. Brandt, Physical Review B 63, 094426 (2001).

Y. Z. Long, Z. H. Yin, and Z. J. Chen, J. Phys. Chem. C 112, 11507 (2008).

V. I. Tsebro, O. E. Omelyanovskii, E. F. Kukovitskii, N. A. Sainov, N. A. Kiselev, and D. N. Zakharov, Journal of Experimental and Theoretical Physics 86, 1216 (1998).

V. P. Arya, V. Prasad, and P. S. A. Kumar, Journal of Physics: Condensed Matter 24, 245602 (2012). 
V. L. Nguyen, B. Z. Spivak, and B. I. Shklovskii, Zh. Eksp. Teor. Fiz. 89, 1770 (1985). A. J. Hoffman, L. Alekseyev, S. S. Howard, K. J. Franz, D. Wasserman, V. A. Podolskiy, E. E. Narimanov, D. L. Sivco, and C. Gmachl, Nat Mater 6, 946 (2007). J. Guo, H. Gu, H. Wei, Q. Zhang, N. Haldolaarachchige, Y. Li, D. P. Young, S. Wei, and Z. Guo, The Journal of Physical Chemistry C 117, 10191 (2013).

S. H. Lee, T. W. Uhm, Y. G. You, S. W. Kim, S. H. Jhang, U. Dettlaff-Weglikowska, and Y. W. Park, Synthetic Metals 198, 84 (2014). 